\title{
SINGULARITIES AND BORDISM OF $q$-PLANE FIELDS AND OF FOLIATIONS ${ }^{1}$
}

\author{
BY ULRICH KOSCHORKE
}

Communicated by Glen E. Bredon, January 3, 1974

1. Introduction. Let $\mathfrak{P N}_{n}(q)$ (resp. $\mathfrak{P N}_{n}^{o r}(q)$ ) be the bordism group of $n$-dimensional smooth manifolds with arbitrary (resp. oriented) $q$-plane fields, and let $\mathfrak{P} \Omega_{n}(q)$ and $\mathfrak{P} \Omega_{n}^{o r}(q)$ denote the corresponding groups based on oriented manifolds. In this paper we present a method which allows us in many cases to determine these groups. We use the forgetful homomorphism $f_{\mathfrak{P}}: \mathfrak{P N}_{n}(q) \rightarrow \mathfrak{N}_{n}(B O(q))$ (resp. $f_{\mathfrak{P}}: \mathfrak{P N}_{n}^{o r}(q) \rightarrow \mathfrak{N}_{n}(B S O(q))$, resp. $\left.f_{\mathfrak{P}}: \mathfrak{P} \Omega_{n}^{(\text {or) }}(q) \rightarrow \Omega_{n}(B(S) O(q))\right)$, which assigns to the bordism class of a $q$-plane field the bordism class of (a classifying map of) the underlying vector bundle. Our point of departure is the following observation. If $\xi$ is a $q$-dimensional vector bundle over an $n$-manifold $M$ and $n \geqq 2 q-3$, then it is always possible to find a vector bundle homomorphism $h: \xi \rightarrow T M$ which is injective outside of a $(q-1)$-dimensional submanifold $S$ of $M$, and such that the kernel of $h$ is 1-dimensional at every point of $S$. We investigate the behavior of $h$ at such a singularity and obtain criteria as to when it is possible to cancel $S$ without getting out of the original bordism class.

If $M$ is closed and $\xi$ is isomorphic to a $q$-dimensional subbundle of $T M$, then the element $T M-\xi$ in the $K$-theory of $M$ can be represented by an $(n-q)$-dimensional bundle, and hence the class $[M, \xi]$ in the bordism of $B(S) O$ satisfies the following vanishing condition:

(V) all Whitney numbers of $[M, \xi]$ containing some $w_{i}(T M-\xi)$, $i>n-q$, as a factor, vanish.

Conversely we obtain

THEOREM 1. Let $n>2 q-2$. Then under all four orientedness assumptions $[M, \xi]$ lies in the image of $f_{\mathfrak{P}}$ if and only if condition $(\mathrm{V})$ is satisfied. Furthermore, the kernel as well as the cokernel of $f_{\mathfrak{P}}$ are finite groups consisting entirely of elements of order 2.

A stable version of the first statement for the case of $\mathfrak{N}_{n}(B O(q))$ has previously been obtained by $\mathbf{R}$. Stong [11] by other methods.

\footnotetext{
AMS (MOS) subject classifications (1970). Primary 57D25, 57D30, 57D90, 58A30; Secondary 55G35, 57D45.

${ }^{1}$ Research partially supported by NSF Grant GP-38215.
} 
COROLLARY 1. $\mathfrak{P N}_{n}(q)$ and $\mathfrak{P N}_{n}^{\text {or }}(q)$ are finite vector spaces over $\boldsymbol{Z}_{2}$. $\mathfrak{P} \Omega_{n}(q)$ and $\mathfrak{P} \Omega_{n}^{o r}(q)$ are finitely generated groups whose torsion consists entirely of elements of order 2 or possibly 4.

These results can be sharpened in many cases to give a complete description of our groups. For example

THEOREM 2. $f_{\mathfrak{P}}$ gives an isomorphism between $\mathfrak{P N}_{n}(q)$ and the subgroup of $\mathfrak{N}_{n}(B O(q))$ consisting of all elements $[M, \xi]$ which satisfy condition (V) above.

For a determination of the plane field bordism groups with other orientedness assumptions see also [6] for $q=1$ and [7] for $q=2$.

If we also take vanishing conditions for the Pontrjagin numbers into account we may in many cases avoid the restriction $n>2 q-2$. This can be done either by also considering singularities with higher dimensional kernel, or by applying our approach to complementary $(n-q)$-plane fields. Thus, e.g., Corollary 1 and Theorem 2 turn out to hold whenever $0 \leqq q \leqq n$, the latter as a consequence of the following duality result.

THEOREM 3. If $0 \leqq q \leqq n$, there is a natural isomorphism $\mathfrak{P N}_{n}(q) \cong$ $\mathfrak{P N}_{n}(n-q)$ obtained by taking complements.

This is not a priori obvious since the standard bordism relation for $q$ plane fields induces a different (stabilized) bordism relation for the complementary $(n-q)$-plane fields.

Next define $\mathfrak{F} \mathfrak{N}_{n}(q)$, $\mathfrak{F} \mathfrak{N}_{n}^{o r}(q)$, $\mathfrak{F} \Omega_{n}(q)$ and $\mathfrak{F} \Omega_{n}^{o r}(q)$ to be the bordism groups of closed $n$-manifolds with smooth $q$-codimensional foliations, satisfying the indicated (co)-orientedness conditions. For $q \geqq 2$ Thurston [13] has shown recently that a foliation on a compact manifold $M$ is essentially given by an $(S) \Gamma$-structure $\gamma$ on $M$ (in the sense of Haefliger [3]) together with a bundle monomorphism from the normal bundle $v(\gamma)$ of $\gamma$ into $T M .^{2}$ Thus when we compare our foliation bordism groups with the corresponding usual bordism groups of Haefliger's classifying spaces $B \Gamma(q)$ and $B S \Gamma(q)$, we are only confronted with a plane field problem and can apply our approach. We obtain for the forgetful homomorphism $f_{\mathfrak{F}}: \mathfrak{F}_{n}^{(o r)}(q) \rightarrow \mathfrak{N}_{n}(B(S) \Gamma(q))$, resp. $f_{\mathfrak{F}}: \mathfrak{F} \Omega_{n}^{(o r)}(q) \rightarrow \Omega_{n}(B(S) \Gamma(q)):$

THEOREM $1^{\prime}$. Let $q \geqq 2$ and $n>2 q-2$. Then under all four orientedness assumptions, an element $[M, \gamma]$ of the $n$-dimensional bordism group of $B(S) \Gamma(q)$ lies in the image of $f_{\mathfrak{F}}$ if and only if the vanishing condition (V) is satisfied by the normal bundle $\xi=v(\gamma)$. Furthermore the kernel as well as the cokernel of $f_{\mathfrak{z}}$ are finite groups consisting entirely of elements of order 2.

${ }^{2}$ ADDED IN PROOF. More recent work of Thurston implies that the results of this paper still hold for foliations of codimension $q=1$. 
This contrasts with the fact that the foliation bordism groups themselves need not even be countably generated. E.g., $\mathscr{F} \Omega_{2 q+1}^{o r}(q)$ surjects onto $\boldsymbol{R}$ for even positive $q$ (see [14]).

THEOREM $2^{\prime}$. If $q \geqq 2$ and $n \geqq 2 q-2$, then $f_{\mathfrak{F}}$ gives an isomorphism between $\mathfrak{F}_{n}(q)$ and the subgroup of $\mathfrak{N}_{n}(B \Gamma(q))$ consisting of all elements $[M, \gamma]$ for which the normal bundle $\xi=v(\gamma)$ satisfies condition $(\mathrm{V})$.

As a corollary to the proof we have

THEOREM 4. For $q \geqq 1, n \geqq 2 q-2$, every $q$-plane field on a closed $n$-manifold is bordant (in $\mathfrak{P N}_{n}(q)$ ) to one which is transversal to a foliation of codimension $q$.

The case $q=1$ (where Thurston's results are not available ${ }^{3}$ ) was settled in [8] by an explicit construction of enough foliations to generate $\mathfrak{P N}_{n}(1)$ by their normal linefields.

Finally, note that the singularity approach can also be fruitfully applied to the bordism of manifolds with tangent $q$-frames, or to the bordism of immersions and, more generally, of $k$-mersions. More details on this point will appear elsewhere (see also [9]).

I would like to thank Peter Landweber for many helpful references.

2. The singularity isomorphism. Let $\mathfrak{N}_{n}(B O(q), \mathfrak{P})\left(\operatorname{resp} . \mathfrak{N}_{n}(B \Gamma(q)\right.$, $\mathfrak{F})$ ) be the bordism group of triples $\left(M, \xi, h^{\prime}\right)\left(\right.$ resp. $\left.\left(M, \gamma, h^{\prime}\right)\right)$ where $M$ is a compact smooth $n$-manifold, $\xi$ is a $q$-plane bundle over $M$ (resp. $\gamma$ is a $\Gamma(q)$-structure on $M$, and we write $\xi$ for its normal bundle $\nu(\gamma))$, and $h^{\prime}: \xi \mid \partial M \rightarrow T(\partial M)$ is a bundle monomorphism. Denote the normal bundle map from $\mathfrak{N}_{n}(B \Gamma(q)$, $)$ into $\mathfrak{N}_{n}(B O(q), \mathfrak{P})$ by $\boldsymbol{v}_{*}$.

Now for $0 \leqq p \leqq q$ consider the $p \cdot(n-q+p)$-codimensional submanifold $A_{p}$ of the total space of the homomorphism bundle $\operatorname{Hom}(\xi, T M)$ where $A_{p}=\bigcup_{x \in M} A_{p}(x)$ and $A_{p}(x)=\left\{g: \xi_{x} \rightarrow T_{x} M \mid g\right.$ linear, $\operatorname{dim}(\operatorname{ker} g$ ) $=p\}$ (cf. [5, p. 120]). If $n \geqq 2 q-3$, or equivalently, if $2(n-q+2)>n$, then, by transversality we can extend $h^{\prime}$ to a vector bundle morphism $h: \xi \rightarrow T M$ which, as a section in $\operatorname{Hom}(\xi, T M)$, goes entirely into $A_{0} \cup A_{1}$ and intersects $A_{1}$ transversally. Denote by $S$ the closed $(q-1)$-dimensional submanifold $h^{-1}\left(A_{1}\right)$ of the interior of $M$. Since $h \mid S$ has constant rank, there are canonical vector bundles Ker, Coker, and Im over $S$ of dimension 1, $n-q+1$, and $q-1$, respectively, where e.g., the fiber of Ker at $x \in S$ is the kernel of $h_{x}: \xi_{x} \rightarrow T_{x} M$. These bundles are related to $\xi|S, T M| S$ and the

\footnotetext{
${ }^{3}$ See footnote 2 .
} 
normal bundle $\nu(S, M)$ of $S$ in $M$ by the following isomorphisms (which are canonical up to homotopy)

$$
\xi \mid S \cong \mathbf{I m} \oplus \mathbf{K e r},
$$

$$
T M \mid S \cong \mathbf{I m} \oplus \text { Coker, }
$$$$
v(S, M) \cong \text { Hom(Ker, Coker); }
$$

and consequently

$$
\text { i: } \mathbf{I m} \oplus \text { Coker } \cong T S \oplus \operatorname{Hom}(\text { Ker, Coker }) .
$$

Associating the bordism class of ( $S$, Ker, Coker) to the class of $\left(M, \xi, h^{\prime}\right)$, we obtain a well-defined homomorphism

$$
\begin{aligned}
\sigma: \mathfrak{N}_{n}(B O(q), \mathfrak{P}) & \rightarrow \mathfrak{N}_{q-1}(B O(1) \times B O(n-q+1)) \\
& \simeq \mathfrak{N}_{q-1}(B O(1) \times B O(q)),
\end{aligned}
$$

provided $n \geqq 2 q-2$. Similarly $\sigma$ is defined on the relative bordism groups $\mathfrak{N}_{n}(B S O(q), \mathfrak{P})$ and $\Omega_{n}(B(S) O(q), \mathfrak{P})$ corresponding to the other orientation cases.

We will say that an element $x=[S, \zeta, \eta]$ of $\mathfrak{N}_{a-1}(B O(1) \times B O(q))$ satisfies condition $O_{b}$ (resp. $\left.O_{m}\right)$ for $(n, q)$ if all those Whitney numbers vanish which either involve $w_{1}(S)+(n-q) w_{1}(\zeta)$ as a factor or which are made up entirely by a positive number of factors of the form $n \cdot w_{2 k}(S)^{2}$ or $n \cdot w_{2 k}(\eta)^{2}, k \geqq 0$ (resp. if all Whitney numbers of $x$ involving $w_{1}(S)+$ $(n-q+1) w_{1}(\zeta)+w_{1}(\eta)$ vanish $)$.

THEOREM 5. Let $n>2 q-2$. Then under all four orientedness assumptions $\sigma$ is an isomorphism into $\mathfrak{N}_{q-1}(B O(1) \times B O(q))$. An element $x$ of

$$
\mathfrak{N}_{q-1}(B O(1) \times B O(q))
$$

lies in the image of $\mathfrak{N}_{n}(B O(q), \mathfrak{P})\left(\right.$ resp. $\mathfrak{N}_{n}(B S O(q), \mathfrak{P})$, resp. $\Omega_{n}(B O(q), \mathfrak{P})$, resp. $\left.\Omega_{n}(B S O(q), \mathfrak{P})\right)$ under $\sigma$ if and only if $x$ is arbitrary (resp. $x$ satisfies condition $O_{b}$, resp. $O_{m}$, resp. $O_{b}$ and $O_{m}$, for $(n, q)$ ).

If in addition $q \geqq 2$, then in all four orientedness cases $\sigma \circ \nu_{*}$ is also an isomorphism onto the image of $\sigma$.

In particular, for fixed $q$ the relative bordism groups of a given orientation type depend only on the parity of $n$.

In the proof we use generalized surgery with core manifolds of dimension $q$ or 1 or 2 . The construction extends to the case of $\Gamma$-structures since the normal bundle map $v: B \Gamma(q) \rightarrow B O(q)$ has a $q$-connected homotopic fiber [3]. 
The relevance of Theorem 5 stems from the following commutative diagram and its analogues in the other orientation cases

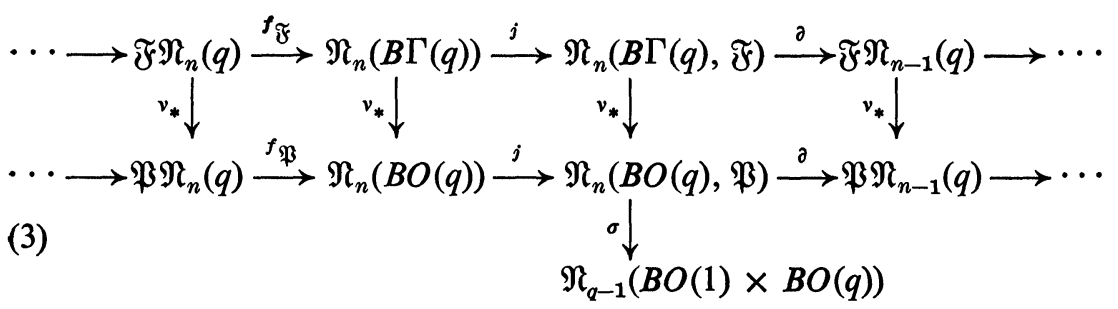

Here the forgetful homomorphisms $j$ and $\partial$ make the horizontal sequences exact.

In order to describe $\sigma \circ j$ in terms of Whitney numbers, assume $M$ to be closed in the discussion above. In a Whitney number of $(S, \mathrm{Ker}$, Coker) eliminate first $w(S)$, and then $w($ Coker), using (1) and (2), and apply the identity

$$
\begin{aligned}
w_{1}(\mathbf{K e r})^{k} \cdot\left(w(T M-\xi)^{\alpha} w(M)^{\beta} \mid S\right)[S] & \\
& =w_{n-\alpha+1+k}(T M-\xi) w(T M-\xi)^{\alpha} w(T M)^{\beta}[M],
\end{aligned}
$$

where $\alpha, \beta$ are multi-indices.

Now Theorem 5 implies Theorem 1, Corollary 1 and Theorem 1'. To obtain a full description of the bordism groups of $q$-plane fields, it remains only to determine the image of $j$, or equivalently, of $\sigma \circ j$ (and to check for possible 4-torsion in $\left.\mathfrak{P} \Omega_{n}^{(o r)}(q)\right)$. For example, a geometric construction yields

THEOREM 6. For $n \geqq 2 q-2$, the homomorphism $\sigma \circ j: \mathfrak{N}_{n}(B O(q)) \rightarrow$ $\mathfrak{N}_{\alpha-1}(B O(1) \times B O(q))$ is onto.

Thus, if no orientation conditions are imposed, the lower horizontal line in diagram (3) breaks down into short exact sequences $(\partial=0)$, and so does the upper line since the middle homomorphism $v_{*}$ is surjective here (compare [1]). This proves Theorems 2 and $2^{\prime}$. Theorem 4 , or equivalently, the surjectivity of the left hand homomorphism $v_{*}$, follows immediately.

\section{REFERENCES}

1. R. Bott and J. Heitsch, $A$ remark on the integral cohomology of $B \Gamma_{a}$, Topology 11 (1972), 141-146. MR 45 \#2738.

2. P. E. Conner and E. E. Floyd, Differentiable periodic maps, Ergebnisse der Math. und ihrer Grenzgebiete, N.F., Band 33, Academic Press, New York; Springer-Verlag, Berlin, 1964. MR 31 \#750.

3. A. Haefliger, Homotopy and integrability, Manifolds-Amsterdam 1970 (Proc. Nuffic Summer School), Lecture Notes in Math., vol. 197, Springer-Verlag, Berlin, 1971, pp. 133-163. MR 44 \#2251. 
4. W. Iberkleid, Splitting the tangent bundle, Thesis, Rutgers University, New Brunswick, N.J., 1973.

5. U. Koschorke, Infinite dimensional K-theory and characteristic classes of Fredholm bundle maps, Proc. Sympos. Pure Math., vol. 15, Amer. Math. Soc., Providence, R.I., 1970, pp. 95-133. MR 43 \#5559.

6. - Concordance and bordism of line fields, Invent. Math., 1974.

7. - Bordism of plane fields and of foliations, Proc. Brasil. Math. Colloq. in Pocos de Caldas, 1973.

8. - Line fields transversal to foliations, Proc. Sympos. Pure Math., vol. 27, Amer. Math. Soc., Providence, R.I. (to appear).

9. - Bordism of immersions and $k$-mersions, Notices Amer. Math. Soc. 21 (1974), A-17. Abstract \#74T-G12 and A405. Abstract \#713-G5.

10. F. P. Peterson, Lectures on cobordism theory, Lectures in Math., Dept. of Math., Kyoto University, 1, Kinokuniya Book Store, Tokyo, 1968. MR 38 \#2792.

11. R. Stong, Subbundles of the tangent bundle, 1972 (preprint).

12. - Relations among characteristic numbers. I, Topology 4 (1965), 267-281. MR 33 \#740.

13. W. Thurston, The theory of foliations of codimension greater than one, 1973 (preprint).

14. - Continuous variation of the Godbillon-Vey invariant in higher codimensions, Lecture held at the Foliations Seminar at the Institute for Advanced Study, Princeton, N.J., 1973.

Department of Mathematics, Rutgers University, New Brunswick, New JERSEY 08903 